\title{
Imaging in Hepatocellular Carcinoma: Guidelines and Future Prospects
}

\author{
Carlo Bartolozzi Valentina Battaglia \\ Diagnostic and Interventional Radiology, University of Pisa, Italy
}

\section{Keywords}

Liver · Cirrhosis · Diagnosis ·

Hepatocellular carcinoma, HCC · Magnetic resonance, MR

\section{Summary}

Background: Non-invasive diagnosis of hepatocellular carcinoma (HCC) in cirrhosis is nowadays the option of choice, as reported by both Western and Eastern guidelines. Methods: Currently, the diagnosis is still focused on the detection of the typical, neoplastic, vascular pattern, characterized by lesion hypervascularization in the arterial phase and by wash-out in the late vascular phase, by means of multidetector computed tomography (MDCT) or magnetic resonance (MR) imaging. In cases of atypical nodules, Western international guidelines suggest, depending on the cases, to perform a second imaging examination, but not contrast-enhanced ultrasound (CEUS), or biopsy tissue sampling. On the contrary, Eastern guidelines have additionally included RES(reticuloendothelial system)-targeted ultrasound or MR examination or hepatobiliary MR imaging for these difficult cases. Results: On the basis of new findings regarding the histological changes occurring in the pathway towards nodular malignancy, new hepatospecific contrast agents are routinely applied in MR studies, allowing to explore cellular metabolic function also in atypical hypoperfused nodules. Conclusions: In the near future, hepatobiliary MR imaging with hepatocyte-selective contrast agents might be considered the best choice to classify preneoplastic, low-risk lesions or atypical nodules with a high potential of malignancy.

\author{
Schlüsselwörter \\ Leber · Zirrhose · Diagnose . \\ Hepatozelluläres Karzinom, HCC · Magnetresonanz, MR
}

\section{Zusammenfassung}

Hintergrund: Die nichtinvasive Diagnose des hepatozellulären Karzinoms (HCC) in Zirrhose stellt heutzutage die erste Behandlung der Wahl dar, wie sowohl in westlichen als auch in östlichen Leitlinien berichtet wird. Methoden: Derzeit fokussiert sich die Diagnose mittels Multidetektor-Computertomographie (MDCT) oder Magnetresonanztomographie (MRT) nach wie vor auf die Detektion des typischen, neoplastischen, vaskulären Musters, das durch Hypervaskularisation von Läsionen in der arteriellen Phase und durch "wash-out» in der späten vaskulären Phase gekennzeichnet ist. Bei atypischen Knoten schlagen die westlichen internationalen Leitlinien je nach Fall eine zweite bildgebende Untersuchung ohne kontrastverstärkten Ultraschall (CEUS) oder eine Stanzbiopsie vor. Im Gegensatz hierzu haben die östlichen Leitlinien RES/retikuloendotheliales System)-spezifisches Ulltraschall- oder MR-Kontrastmittel oder die hepatobiliäre MRT aufgenommen. Ergebnisse: Auf der Grundlage der neuen Erkenntnisse bezüglich der histologischen Veränderungen, die im Verlauf der Entstehung von malignen Knötchen auftreten, werden neue hepatospezifische Kontrastmittel routinemäßig in MRT-Studien angewandt; dies erlaubt die Erforschung der zellulären metabolischen Funktion auch in atypischen Knoten. Schlussfolgerungen: In der nahen Zukunft könnte sich die hepatobiliäre MRT mit Hepatozyten-spezifischen Kontrastmitteln als Methode der Wahl für präneoplastische Läsionen mit niedrigem Risiko und atypische Knoten mit hohem Risiko für Malignität etablieren.

\section{KARGER \\ Fax +497614520714 \\ Information@Karger.com}

www.karger.com (c) 2013 S. Karger GmbH, Freiburg

$1662-6664 / 13 / 0292-0072 \$ 38.00 / 0$

Accessible online at:

www.karger.com/vim
Prof. Carlo Bartolozzi, MD

Diagnostic and Interventional Radiology, University of Pisa

Via Paradisa 2, 56100 Pisa, Italy

carlo.bartolozzi@med.unipi.it 


\section{Introduction}

Hepatocellular carcinoma ( $\mathrm{HCC}$ ) is one of the most common malignant neoplasms worldwide, with an estimated incidence of more than 500,000 new cases per year.

The most common background in which the neoplasm arises is represented by cirrhosis. In Western countries cirrhosis is mainly due to chronic infection caused by hepatitis $\mathrm{C}$ virus (HCV), followed by chronic hepatitis B virus (HBV) infection and alcohol abuse; other causes of chronic liver injury frequently related to the development of HCC are represented by cholestatic, autoimmune, and metabolic disorders as well as nonalcoholic steatohepatitis (NASH) [1,2].

Clinically, HCC does not show a specific presentation as the symptoms of the underlying cirrhosis are frequently dominant. As a consequence, scheduled surveillance of selected patients is currently the major practical approach for an early diagnosis of a neoplasm, as suggested in all of the international guidelines [3-5].

\section{HCC Screening}

Algorithms proposed by different guidelines, which might slightly differ in terms of target screening population as well as management of small detected nodules, have been employed to assist in the detection of small liver nodules found during surveillance.

The recently updated guidelines, issued by the American Association for the Study of Liver Diseases (AASLD), identify those affected by cirrhosis or by NASH as well as those affected by chronic HBV or HCV infection, even without an evidence of cirrhosis, as high-risk patients [4]. While a different surveillance management of HBV patients was suggested in the past, nowadays the time interval for surveillance reported by Western international guidelines, i.e. AASLD and EASL/EORTC (European Association for the Study of the Liver and European Organization for Research and Treatment of Cancer), is 4-6 months, independently of the cause of liver damage, since retrospective studies have demonstrated no benefit from a closer follow-up in terms of survival $[3,4,6,7]$.

On the contrary, Eastern guidelines, issued by the Asian Pacific Association for the Study of the Liver (APASL), indicate as appropriate an ultrasound (US) follow-up, performed every 3 months [5].

Selected patients do benefit from a prospective screening as it increases the possibility for early diagnosis of a neoplasm, allowing access to several, personalized, therapeutic options $[8,9]$.

In the past few years, both US examination and measurement of alpha-fetoprotein (AFP) levels were considered as the two main screening tools in the surveillance of cirrhotic patients.
Several studies have shown the limited role of AFP because of its poor specificity and low positive predictive value (PPV) in assessing the presence of a neoplasm specifically in a low-prevalence population such as in Western Europe; as a result, measurement of AFP has been removed from Western guidelines $[10,11]$.

Again, US, because of its low cost, its widely spread availability, and the absence of related risks, is considered as the unique and most appropriate approach for screening cirrhotic patients, showing otherwise a wide range of sensitivity (ranging between 58 and $89 \%$ ), mainly related to the skills of the operator [12].

On the contrary, multidetector computed tomography (MDCT) and magnetic resonance (MR) imaging, despite their high reliability in diagnostic assessment, are not recommended as screening tools because of their sustained costs and possibly related harms such as radiation exposure and adverse events of contrast agents.

\section{HCC Diagnosis}

The development of a neoplasm in cirrhosis is a long-lasting process that might take months or even years. The continuous inflammatory stimulation which is caused by chronic $\mathrm{HCV} / \mathrm{HBV}$ infections determines a chronic injury of hepatocytes and stromal liver cells. Many asynchronous cellular changes occur along the pathway from normal hepatocytes to neoplastic cells so that different types of hepatocellular nodules can be contemporarily present in a cirrhotic parenchyma: regenerative nodules, low-grade dysplastic nodules (LGDN), high-grade dysplastic nodules (HGDN), early HCC and, finally, overt HCC.

Particularly, HGDN nodules as well as early HCC are considered as premalignant and early malignant nodules since foci of HCC can be found inside HGDNs, while in cases of early HCC, usually all typical vascular changes have not yet occurred, unless the cells show signs of malignant degeneration $[13,14]$.

On the contrary, a decrease of portal venous supply in overt HCC (although still present only in very well differentiated lesions) and the development of neoangiogenesis, characterized by the presence of unpaired arteries and consistent arteriovenous shunts, is well established [14].

On the basis of such peculiar, microscopic, vascular architecture, non-invasive diagnosis of HCC in liver cirrhosis is actually demanded to perform post-contrastographic, dynamic imaging techniques, which are considered as the standard of reference.

Diagnostic MDCT should be performed by examining at least three phases (arterial, portal venous, and late phase) and by fixing the acquisition timing after contrast injection. Depending on the necessity to analyze arterial feeders as well as to obtain a vascular mapping of the liver, an early arterial phase should also be included in the protocol [15]. 


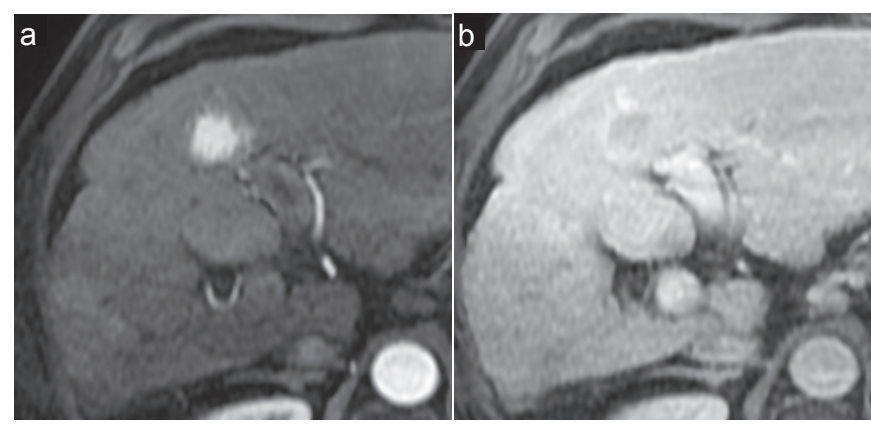

Fig. 1. Typical HCC enhancement pattern during dynamic MR study. Nodule within segment IV shows (a) a clear-cut enhancement on arterial phase and (b) a wash-out in the late vascular phase.

Similarly, in a standard post-contrastographic, dynamic MR study, performed with a high-field scanner (1.5T-3T), the same three vascular phases should be acquired. Due to the high temporal resolution of the system, a continuous acquisition after contrast agent injection is frequently carried out, independently of a fixed timing [16].

The so-called typical neoplastic pattern, characterized by a wash-in of early lesions in the arterial phase and a wash-out in the portal venous/late phases, has shown a PPV of $100 \%$ for a diagnosis of neoplasm, independently of the dimensions and grading of the lesions (fig. 1) [17, 18].

It should be noted that none of the guidelines reports which cross-sectional imaging modality between MDCT and MR should be applied in order to evaluate a suspected nodule detected during US surveillance; however, it is strongly recommended to perform both examinations in the case of lesions $<2 \mathrm{~cm}$ by following dedicated acquisition protocols, possibly at specialized centers [4]. The EASL/EORTC guidelines recommend that only in centers of excellence with 'high-end radiological equipment' the assessment of nodules $<2 \mathrm{~cm}$ should be performed with one imaging modality only $[3,4]$. In case of doubt a biopsy must follow.

Dynamic MR and MDCT studies show a similar sensitivity in the detection of a typical, neoplastic, vascular pattern ranging between 44 and $62 \%$ as well as between 44 and $53 \%$, respectively, although MR has been proven to be superior in assessing a correct diagnosis, especially in nodules smaller than $2 \mathrm{~cm}$ [19].

The latest EASL/EORTC as well as the AASLD guidelines have removed contrast-enhanced ultrasound (CEUS) from the diagnostic algorithm of HCC since it does not reliably differentiate between HCC and cholangiocellular carcinoma, showing identical wash-out phenomena; nevertheless, due to the high sensitivity of the technique in the detection of microcirculation, such a method can be suggested as a method of problem solving in suspected cases not showing hypervascularization in the arterial phase during MDCT or MR, especially in nodules smaller than $2 \mathrm{~cm}$ (fig. 2).

Moreover, CEUS evaluation, carried out during US examination, can be helpful in the differential diagnosis of other liver nodules that might be present in cirrhotic livers, such as
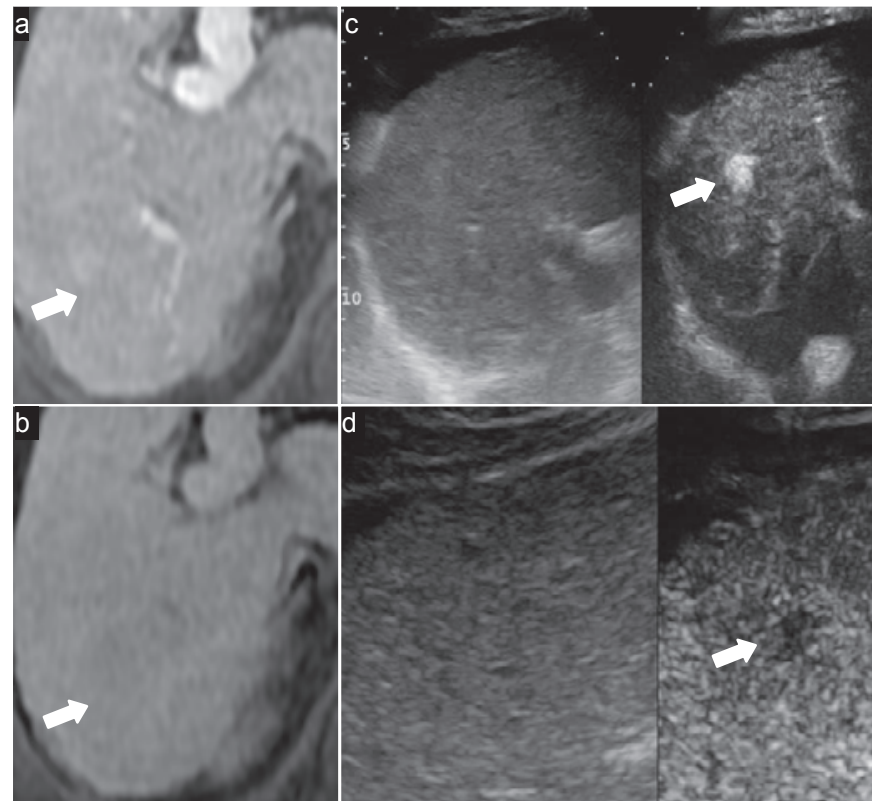

Fig. 2. Usefulness of CEUS. a At MR examination, a nodule (white arrow) in the arterial phase does not show a clear-cut enhancement. b In the late vascular phase, it shows as mildly hypointense. c,d However, at CEUS examination, performed 10 days after MR, the nodule is (c) typically hypervascular in the arterial phase and (d) hypoechoic in the late phase.

hemangiomas, which are immediately characterized and ruled out from a subsequent, diagnostic work-up [20].

If the typical hallmarks of HCC at dynamic imaging are approved by all current guidelines, significant differences in the management of detected nodules at US surveillance on the basis of the dimensions of the nodules are appreciable among different diagnostic algorithms.

Regarding the EASL guidelines, the threshold of evaluating detected nodules by means of a single imaging technique is in fact $2 \mathrm{~cm}$, while smaller nodules (between 1 and $2 \mathrm{~cm}$ ) should be investigated by two imaging modalities. Nodules smaller than $1 \mathrm{~cm}$ should undergo a scheduled follow-up by US instead [3].

On the contrary, the AASLD guidelines suggest that the detection of the typical enhancement pattern at one single imaging modality in nodules larger than $1 \mathrm{~cm}$ can be considered as sufficient to formulate a diagnosis of neoplasm [4].

Finally, eastern guidelines disregard the dimensions of the nodules because the diagnosis of malignancy could be assessed even in lesions smaller than $1 \mathrm{~cm}$ in the case of a typical enhancement pattern during the dynamic imaging evaluation [5].

Since only two-thirds of HCCs are reported to show a typical pattern, the guidelines have furthermore focused on the attention concerning atypical nodules, which are hypovascular in the arterial phase, presenting again striking differences in management $[2,21]$.

While both EASL/EORTC and AASLD guidelines, focusing strongly on the dynamic pattern of the nodules, suggest biopsy as the third diagnostic step in the case of atypical nod- 
Fig. 3. Baseline MR examination. On baseline sequence, the small HCC nodule shows (a) as hyperintense on an in-phase T1-weighted image because of glycogen content, while on out-phase (b), the anterior part is hypointense due to the fat component within the lesion. Hyperintensity of the nodule on baseline T2weighted image (c) is due to hypercellularity.
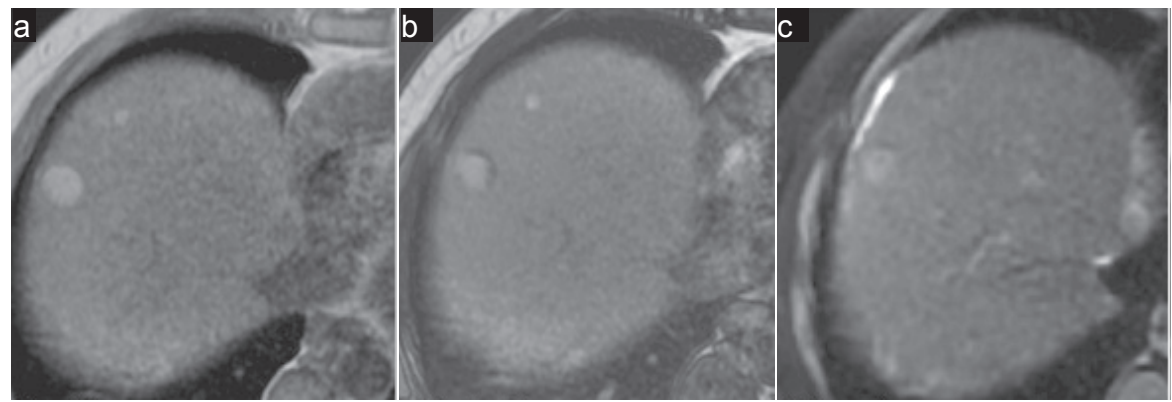

Fig. 4. Baseline and post-contrastographic examination of an atypical nodule within cirrhosis. The nodule (white arrow) shows (a) as hyperintense on baseline T2-weighted image, (b) as hypointense on baseline T1weighted image, (c) a restriction of diffusivity at DWI (b, 800), and $(\mathbf{e}, \mathbf{f})$ as hypointense in the late dynamic and hepatobiliary phase. Despite all imaging findings fit in with a diagnosis of HCC, the lack of hypervascularization in the arterial phase (d) does not allow a diagnosis of neoplasm.

Biopsy established a diagnosis of HGDN.
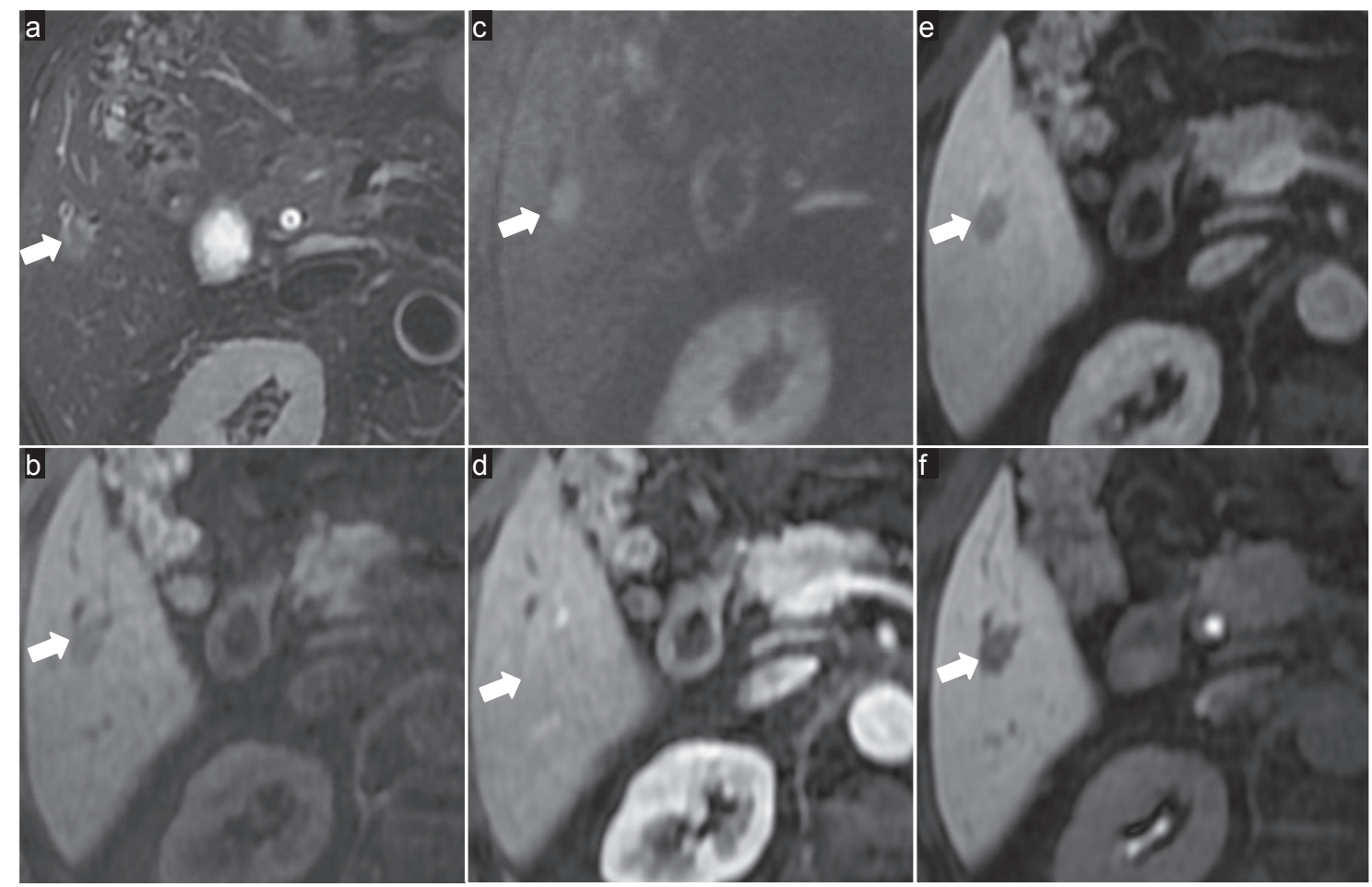

ules larger than $1 \mathrm{~cm}$, the APASL and the Japanese guidelines have introduced new diagnostic tools.

Particularly, hypovascular nodules should be investigated regardless of size by applying RES- or hepatocyte-specific contrast agents during MR examinations or CEUS using Sonazoid [5].

Nevertheless, all of the guidelines recommend biopsy as a solving tool and a third diagnostic step in cases in which all previous examinations did not lead to a definite diagnosis of the nature of the nodules.

Both EASL/EORTC and AASLD guidelines recommend the use of percutaneous sampling in cases of atypical nodules larger than $1 \mathrm{~cm}$ at the end of the diagnostic algorithm, while the APASL guidelines suggest performing the procedure independently of the size of the lesions.

However, it should be considered that biopsy, affected by the risks of bleeding and seeding, might lead to a misdiagnosis in approximately $30 \%$ of the cases, mainly because of false negative results due to wrong sampling, especially in small tu- mors. This represents a non-negligible drawback of a method that is generally considered as a solving tool [21].

\section{New Prospectives}

Despite being very specific, the diagnosis of HCC based on the detection of the sole neoangiogenesis has shown some limitations regarding its sensitivity. Emphasized by new technological improvements, other diagnostic elements have therefore been considered [17].

Due to its intrinsic capability to assess the components of lesions also according to baseline acquisitions, such as glycogen, hemorrhage, water, or metals, and to highlight pathologic changes taking place during the carcinogenetic process, the role of MR in cirrhosis has increased continuously (fig. 3).

In fact, MR should be considered as a multiparametric technique which enables to give a paramount overview of all data that might increase the diagnostic confidence. 
Two main MR tools seem to be applicable in the diagnostic work-up, i.e. diffusion-weighted imaging (DWI) and hepatocyte-specific contrast agents (fig. 4).

\section{Diffusion-Weighted Imaging}

DWI is a dedicated MR sequence which allows for the evaluation of the random motion (related to thermal effects) of water molecules ('Brownian motion') within biological tissues due to not being influenced by magnetic field strength, perfusion, or microcirculation. Its application in the abdominal district, once limited, has been possible due to the development of ultrafast sequences such as echoplanar imaging that enable the acquisition of the entire volume in a single breathholding (30-60 s per image), with a reduction of motion artefacts.

Recently, DWI has been introduced in liver MR protocols as several studies have reported its usefulness in improving the detection and characterization of focal liver lesions, especially by applying a quantitative evaluation of water molecule motion by measuring the apparent diffusion coefficient of the lesions [22].

This technique allows the detection of the restriction of water molecule diffusion within the neoplastic lesions, representing an additional element that might increase the diagnostic suspicion of a neoplasm.

Nevertheless, even if a number of studies have reported on the usefulness of DWI in differential diagnosis of benign or malignant focal liver, no consistent data are available regarding its usefulness in the differential diagnosis of a nodule within cirrhosis. Therefore, no guideline defines its role in the diagnostic algorithm [23, 24].

\section{MR-Hepatospecific Contrast Agents}

As already mentioned, intravascular contrast agents (gadolinium (Gd) chelates) allow a reliable recognition of the presence of neoangiogenesis and vascular rearrangements, which is not present in about $30 \%$ of the HCCs.

Through the progression from regenerative nodule to LGDN, to HGDN, and finally to overt HCC, a main, further parallel process takes place besides vascular alteration, represented by the progressive loss of biliary polarization of the hepatocyte and the derangement of its microscopic, secretory structure.

From a pathological point of view, it has been recently reported that two main categories of anionic, membrane transporters, represented by proteins of the family of OATP (organic anionic transporter protein) and MRP (multidrug-resistance protein), involved in the bilirubine metabolism can be altered. Those carriers are located on the sinusoidal and on the biliary pole of hepatocytes, respectively [25].

Despite no definite data are available regarding the given relationship between loss of membrane proteins and cellular dedifferentiation, especially in the case of HGDNs and early HCCs, it has been reported that membrane carriers are altered or not functioning regularly in neoplastic nodules [25].
However, both of these two preneoplastic conditions need to be carefully evaluated for a correct patient management [26].

The recent introduction of hepatobiliary contrast agents in MR studies, and especially of the highly lipophilic compound Gd-EOB-DTPA, has provided an additional tool for the assessment of the metabolic function of nodules. In fact, due to a competitive binding to bilirubine transporters, these agents allow the exploration of the residual performance of specific, hepatocytic, cellular membrane proteins and intracellular metabolic activities [27].

Moreover, these agents enable the evaluation of both dynamic vascular and metabolic nodular functions in a singlesession study since the contrast is taken up within functioning hepatocytes and then excreted at the level of the biliary pole at the end of the intravascular phase. This metabolic phase occurs between 20 and 40 min after the injection; imaging acquisition of such a phase is scheduled at the end of the MR study protocol.

In recent studies, the lack of contrast agent uptake in the hepatobiliary phase has been related to a premalignant (HGDN) or malignant degeneration (early/overt HCC), even in the case of absence of the typical vascular pattern $[18,28]$.

\section{Conclusions}

Diagnosis of HCC still remains a challenging issue, also considering the complex management of the huge number of patients suffering from chronic liver diseases.

Surveillance is entirely focused on US examination and is otherwise affected by some limitations in terms of detection rate and specificity. In order to be characterized, nodules detected during surveillance are subject to a second-step diagnostic tool (MDCT or MR).

Until now, imaging diagnosis relies upon the detection of typical vascular patterns (wash-in/wash-out) during contrastenhanced MDCT or MR. However, even in earlier phases of the carcinogenetic pathway, the necessity to explore different alterations that might suggest cellular dedifferentiation has been stressed.

MR has gained a major role in liver imaging due to its inherent advantages, mainly represented by the possibility to explore intracellular components, and due to new technical developments.

Therefore, a more influential role of MR by adding both DWI as well as hepatobiliary contrast agents in the diagnostic work-up can be foreseen and, consequently, a revision of the current guidelines regarding the diagnostic algorithm of HCC should be taken into consideration.

\section{Disclosure Statement}

The authors have nothing to disclose.
Bartolozzi/Battaglia 


\section{References}

1 Davila JA, Morgan RO, Shaib Y, McGlynn KA El-Serag HB: Hepatitis C infection and the increasing incidence of hepatocellular carcinoma: a population-based study. Gastroenterology 2004;127: 1372-1380.

2 Ascha MS, Hanouneh IA, Lopez R, Tamimi TA, Feldstein AF, Zein NN: The incidence and risk factors of hepatocellular carcinoma in patients with nonalcoholic steatohepatitis. Hepatology 2010;51: 1972-1978.

3 European Association for the Study of the Liver; European Organisation for Research and Treatment of Cancer: EASL-EORTC clinical practice guidelines: management of hepatocellular carcinoma. J Hepatol 2012;56:908-943.

4 Bruix J, Sherman M; American Association for the Study of Liver Diseases: Management of hepatocellular carcinoma: an update. Hepatology 2011;53: 1020-1022.

5 Omata M, Lesmana LA, Tateishi R, et al: Asian Pacific Association for the Study of the Liver consensus recommendations on hepatocellular carcinoma. Hepatol Int 2010;4:439-474.

6 Bruix J, Sherman M; Practice Guidelines Committee, American Association for the Study of Liver Diseases: Management of hepatocellular carcinoma. Hepatology 2005;42:1208-1236.

7 Trevisani F, De NS, Rapaccini G, et al: Semiannual and annual surveillance of cirrhotic patients for hepatocellular carcinoma: effects on cancer stage and patient survival (Italian experience). Am J Gastroenterol 2002;97:734-744.

8 Zhang BH, Yang BH, Tang ZY: Randomized controlled trial of screening for hepatocellular carcinoma. J Cancer Res Clin Oncol 2004;130:417-422.

9 Sherman M: Surveillance for hepatocellular carcinoma and early diagnosis. Clin Liver Dis 2007;11: 817-837.

10 Sherman M: The radiological diagnosis of hepatocellular carcinoma. Am J Gastroenterol 2010;105: 610-612.
11 Singal A, Volk ML, Waljee A, et al: Meta-analysis: surveillance with ultrasound for early-stage hepatocellular carcinoma in patients with cirrhosis. Aliment Pharmacol Ther 2009;30:37-47.

12 Bolondi L: Screening for hepatocellular carcinoma in cirrhosis. J Hepatol 2003:39:1076-1084.

13 Theise ND, Park YN, Kojiro M: Dysplastic nodules and hepatocarcinogenesis. Clin Liver Dis 2002;6: 497-512.

14 International Consensus Group for Hepatocellular Neoplasia: Pathologic diagnosis of early hepatocellular carcinoma: a report of the International Consensus Group for Hepatocellular Neoplasia. Hepatology 2009;49:658-664

15 Zech CJ, Reiser MF, Herrmann KA: Imaging of hepatocellular carcinoma by computed tomography and magnetic resonance imaging: state of the art. Dig Dis 2009;27:114-124.

16 Willatt JM, Hussain HK, Adusumilli S, Marrero JA: MR imaging of hepatocellular carcinoma in the cirrhotic liver: challenges and controversies. Radiology 2008;247:311-330.

17 Bartolozzi C, Battaglia V, Bargellini I, et al: Contrast-enhanced magnetic resonance imaging of 102 nodules in cirrhosis: correlation with histological findings on explanted livers. Abdom Imaging 2012; DOI: 10.1007/s00261-012-9952-9.

18 Forner A, Vilana R, Ayuso C, et al: Diagnosis of hepatic nodules $20 \mathrm{~mm}$ or smaller in cirrhosis: prospective validation of the noninvasive diagnostic criteria for hepatocellular carcinoma. Hepatology 2008;47:97-104.

19 Kim K, Kim CS, Han YM, et al: Detection of hepatocellular carcinoma: gadoxetic acid-enhanced 3-dimensional magnetic resonance imaging versus multi-detector row computed tomography. J Comput Assist Tomogr 2009;33:844-850.

20 Dietrich CF: Liver tumor characterization - comments and illustrations regarding guidelines. Ultraschall Med 2012;33(suppl 1):S22-30.
21 Bolondi L, Gaiani S, Celli N, et al: Characterization of small nodules in cirrhosis by assessment of vascularity: the problem of hypovascular hepatocellular carcinoma. Hepatology 2005;42:27-34.

22 Ichikawa T, Cederle MP, Grazioli L, Marsh W: Fibrolamellar hepatocellular carcinoma: pre- and posttherapy evaluation with CT and MR imaging. Radiology 2000;217:145-151.

23 Namimoto T, Yamashita Y, Sumi S, et al: Focal liver masses: characterization with diffusion weighted echo-planar MR imaging. Radiology 1997;204:739-744.

24 Gourtsoyianni S, Papanikolaou N, Yarmenitis S, et al: Respiratory gated diffusion-weighted imaging of the liver: value of apparent diffusion coefficient measurements in the differentiation between mos commonly encountered benign and malignant focal liver lesions. Eur Radiol 2008;18:486-492.

25 Narita M, Hatano E, Arizono S, et al: Expression of OATP1B3 determines uptake of Gd-EOBDTPA in hepatocellular carcinoma. J Gastroenterol 2009;44:793-798.

$26 \mathrm{Yu}$ NC, Chaudhari V, Raman SS, et al: CT and MRI improve detection of hepatocellular carcinoma, compared with ultrasound alone, in patients with cirrhosis. Clin Gastroenterol Hepatol 2011;9:161-167.

27 Kitao A, Zen Y, Matsui O, et al: Hepatocellular carcinoma: signal intensity at gadoxetic acid-enhanced MR imaging - correlation with molecular transporters and histopathologic features. Radiology 2010;256:817-826.

$28 \mathrm{Kim}$ SH, Kim SH, Lee J, et al: Gadoxetic acidenhanced MRI versus triple-phase MDCT for the preoperative detection of hepatocellular carcinoma. AJR Am J Roentgenol 2009;192:1675-1681. 